\title{
Early glomerulopathy is present in young, Type 1 (insulin-dependent) diabetic patients with microalbuminuria
}

\author{
H.-J. Bangstad ${ }^{1}$, R. Osterby ${ }^{2}$, K. Dahl-Jørgensen ${ }^{1}$, K.J. Berg ${ }^{3}$, A.Hartmann ${ }^{3}$, G.Nyberg ${ }^{4}$, S.Frahm Bjørn ${ }^{2}$, \\ K.F.Hanssen ${ }^{1}$ \\ ${ }^{1}$ Aker Diabetes Research Center, Aker University Hospital, Oslo, Norway \\ ${ }^{2}$ Institute of Experimental Clinical Research, Kommunehospitalet, Århus, Denmark \\ ${ }^{3}$ Nephrology Section, Medical Department B, Rikshospitalet, Oslo, Norway \\ ${ }^{4}$ Transplant Unit, Sahlgrenska Hospital, Göteborg, Sweden
}

\begin{abstract}
Summary. Increased urinary albumin excretion, microalbuminuria, may be the first sign of early diabetic nephropathy. We examined glomeruli by morphometric methods in 17 patients with Type 1 (insulin-dependent) diabetes mellitus and microalbuminuria. The median age was 19 (range 18-29) years, duration of diabetes $12(8-15)$ years, mean blood pressure $93(87-115) \mathrm{mm} \mathrm{Hg}$, glomerular filtration rate 132 (101-209) $\mathrm{ml} \cdot \mathrm{min}^{-1} \cdot 1.73 \mathrm{~m}^{2-2}$, albumin excretion rate (mean over 1 year) 32 (15-194) $\mu \mathrm{g} / \mathrm{min}$. Reference data were obtained from 11 healthy kidney donors. Mesangial volume estimates were obtained by serial sectioning in three total profiles in each of three glomeruli in diabetic patients. Basement membrane thickness and matrix volume fraction were estimated from one level per glomerulus. Two matrix parameters, matrix star volume and matrix thickness, were estimated. Interstitial volume fraction in cortex was measured by light microscopy. The morphological parameters were significantly increased in the diabetic group compared to the control group, basement membrane thickness (mean with $95 \%$ confidence intervals) was $595 \mathrm{~nm}(549-641 \mathrm{~nm})$ vs
\end{abstract}

$305 \mathrm{~nm}(287-325 \mathrm{~nm}), p=0.0001$; mesangial volume fraction $0.22(0.21-0.23)$ vs $0.19(0.18-0.21), p=0.04$, and matrix volume fraction $0.13(0.12-0.13$ vs $0.09(0.08-0.10)$, $p=0.001$. Also matrix star volume and thickness, interstitial volume fraction and mean capillary diameter were significantly increased. The intra-individual variation among glomeruli expressed as coefficient of variation was $7.4 \%$ vs $9 \%$ (basement membrane thickness) and $11.7 \%$ vs $25 \%$ (mesangial volume fraction) in the diabetic and the control group, respectively. Increment of basement membrane thickness and matrix volume fraction per year were significantly correlated with mean 1-year $\mathrm{HbA}_{1 \mathrm{c}}(r=0.55$ and $r=0.51$, respectively). We conclude that microalbuminuria in Type 1 diabetes is associated with increased basement membrane thickness and also mesangial matrix expansion. This increment seems to correlate with glycaemic control.

Key words: Diabetic glomerulopathy, microalbuminuria, basement membrane thickness, mesangial expansion, mesangial matrix, stereology, hyperglycaemia.
Microalbuminuria (MA) has been shown to be predictive for later clinical diabetic nephropathy [1-3]. MA is by some investigators regarded as an expression of general vascular disease and a marker of early nephropathy [4]. This is questioned by other authors claiming that microalbuminuria alone, without elevated blood pressure or decreased glomerular filtration rate, does not indicate underlying glomerular lesions different from those in patients with normoalbuminuria [5]. Whether persistent microalbuminuria in Type 1 (insulin-dependent) diabetes mellitus denotes distinct morphological changes in the kidney has been addressed in only a few studies [5-7].

The aim of our study was to quantify morphological glomerular changes in young (age 18-29 years), Type 1 diabetic patients with persistent microalbuminuria and compare the results with those found in non-diabetic subjects. The results are discussed according to recently pub- lished findings in diabetic patients with normoalbuminuria $[8,9]$.

\section{Subjects and methods}

\section{Subjects}

Persistent microalbuminuria was screened for in 371 Type 1 diabetic patients between $10-30$ years of age with diabetes duration of more than 5 years. The patients came from five different out-patient clinics (282 subjects from Aker University Hospital, paediatric and medical departments, and 89 from three nearby paediatric departments). Persistent microalbuminuria was defined as albumin excretion rate between $15-200 \mu \mathrm{g} / \mathrm{min}$ in at least two out of three overnight urine samples during 1 year. Forty-five patients (12\%) fulfilled this criterion. Thirty-three patients consented to participate in a randomized, prospective study concerning the importance of improved blood glucose control in adolescents and young adults. The study in- 
Table 1. Clinical data (median and range)

\begin{tabular}{|c|c|c|}
\hline & $\begin{array}{l}\text { Diabetic } \\
\text { patients }\end{array}$ & $\begin{array}{l}\text { Control } \\
\text { subjects }\end{array}$ \\
\hline$n$ (female/male) & $17(8 / 9)$ & $11(8 / 3)$ \\
\hline Age (years) & $19(18-29)$ & $48(21-58)$ \\
\hline Diabetes duration (years) & $12(8-15)$ & - \\
\hline $\operatorname{GFR}\left(\mathrm{ml} \cdot \mathrm{min}^{-1} \cdot 1.73 \mathrm{~m}^{2-2}\right)$ & $132(101-209)$ & $104(86-125)$ \\
\hline $\mathrm{AER}^{\mathrm{a}}(\mu \mathrm{g} / \mathrm{min})$ & $32(15-194)$ & - \\
\hline $\mathrm{HbA}_{1 \mathrm{c}} \mathrm{a}(\%)$ & $10.5(7.9-12.6)$ & - \\
\hline Systolic blood pressure $(\mathrm{mm} \mathrm{Hg})$ & $120(110-150)$ & $120(110-150)$ \\
\hline Diastolic blood pressure ( $\mathrm{mm} \mathrm{Hg}$ ) & $78(67-98)$ & $80(70-90)$ \\
\hline Mean blood pressure $(\mathrm{mm} \mathrm{Hg})$ & $93(87-115)$ & $93(83-105)$ \\
\hline
\end{tabular}

GFR, Glomerular filtration rate; AER, urinary albumin excretion rate (overnight)

a values are the mean of measurements taken during 1 year

cludes baseline renal biopsies and follow-up biopsies at 2-3 years. This report deals with the glomerular morphology from the first 17 kidney biopsies investigated.

The patients had neither clinical neuropathy nor proliferative retinopathy (three had no retinopathy at all, while 14 had simplex retinopathy with median 23 red spots (microaneurisms and haemorrhages), range $3-187$, by $50^{\circ}$ fundus photography). Sixteen of the patients had normal arterial blood pressure $(<140 / 90 \mathrm{~mm} \mathrm{Hg})$. One was hypertensive (140/97 $\mathrm{mm} \mathrm{Hg}$ ) at the beginning of the study, but the blood pressure decreased to $135 / 85 \mathrm{~mm} \mathrm{Hg}$ during 6 months without treatment. The clinical data are given in Table 1. The protocol was approved by the regional ethics committee and renal biopsy was performed only in patients above 18 years of age after written consent. No complications were experienced.

The control biopsies were taken from 11 living kidney donors who had undergone an extensive investigation prior to nephrectomy to ensure normal renal function [10] (Table 1).

\section{Clinical and laboratory studies}

Urinary albumin excretion rate (AER) was measured in three timed overnight urine samples the year preceding the biopsy. Data expressed as medians with ranges are given in Table 1 . The albumin concentration was measured by immunoturbidimetry in samples kept at $4^{\circ} \mathrm{C}$ from 1 to 3 days. The inter-assay coefficient of variation(CV) was $4.7 \%$ in the range of $10-50 \mathrm{mg} / \mathrm{l}$. Urine samples were negative for leucocytes, nitrite, albumin and ketones as determined by NephurTest ${ }^{\mathrm{R}}+$ Leuco (Boehringer-Mannheim GmbH, Mannheim, FRG).

Glomerular filtration rate (GFR) in the diabetic group was measured by inulin clearance after oral water loading $(>20 \mathrm{ml} / \mathrm{kg}$ ) with subsequent urine osmolality below $200 \mathrm{mosm} / \mathrm{kg}$. High concentration of interfering glucose was removed by glucose oxidase. In the control group the GFR was determined as the plasma clearance of ${ }^{51} \mathrm{Cr}$-EDTA after single bolus injection [11]. The GFRs were corrected for body surface area $\left(1.73 \mathrm{~m}^{2}\right)$.

Haemoglobin $\mathrm{A}_{1 c}\left(\mathrm{HbA}_{1 c}\right)$ was analysed by an HPLC-method using a "Diamat"-machine (Biorad, Richmond, Calif., USA). The normal range is $4.3-6.1 \%$, inter-assay CV $3 \%$. The mean of $3-4$ $\mathrm{HbA}_{1 \mathrm{c}}$-measurements per patient during 1 year is given.

Blood pressure was measured by conventional mercury sphygmomanometer with the patient sitting after 10 min rest. Mean blood pressure in $\mathrm{mm} \mathrm{Hg}$ was calculated as $1 / 3 \times$ systolic $+2 / 3 \times$ diastolic (Korotkoff $V$ ) pressure. Measurements in the diabetic patients represent the mean values of four measurements during a 2-month period.

\section{Renal biopsies}

In the diabeticpatients ultrasound-guidedkidney biopsies were taken with an 18 gauge needle integrated automatic gun-biopsy system (Biopty, Bard Inc, Covington, Ga., USA). The tissue was immediately immersedinto $2 \%$ glutaraldehyde solutioninmodified Tyrode buffer [12] and mailed in this fixative to the laboratory in Arhus. Embeddinginto Vestopal was done after 3-10 days storage (mean6.3 days) in fixative. In the living donors a cortical wedge biopsy was taken immediately after the re-establishment of blood flow in the transplanted kidney. These biopsies were mailed in the fixative ( $2 \%$ glutaraldehyde in cacodylate buffer) and embedded into Vestopal.

\section{Measurement of structural parameters}

Structural quantities relevant to diabetic glomerulopathy were estimated. The structures in question are the peripheral basement membrane $(\mathrm{BM})$, the mesangial regions and the mesangial matrix (Fig. 1). With the main aim of improving the precision in the estimate of mesangial volume fraction we developed the following procedure:

Sampling from the tissue block; sections of $1 \mu \mathrm{m}$ thickness were cut, picked up and stained with toluidine blue for light microscopy. The first section in the block, the baseline section, was not used for sampling. Sequential sections with $10-\mu \mathrm{m}$ intervals were inspected, and any new glomerulus appearing in one of these sections was sampled for analysis. Thin sections were cut at three sequential levels, $60 \mu \mathrm{m}$ and multiples thereof, from the baseline section. With this procedure three glomeruli were sampled independently of size or structure, and ultrastructure was studied in the set of three parallel cross-sections with a random position along the glomerular diameter. The sections stained for light microscopy $(10-\mu \mathrm{m}$ intervals) were used for the determination of glomerular volume. The data presented for the control group were obtained from at least three randomly selected glomeruli [8] using one level with a random position in each glomerulus.

Quanification by electron microscopy: the glomerular profiles with level distance of $60 \mu \mathrm{m}$, were recorded with overlapping micrographs to produce photomontages at a final magnification of $2350 \times$. A minimum of three profiles from each of three glomeruli were obtained. Since large, untrimmed sections were used it was possible in

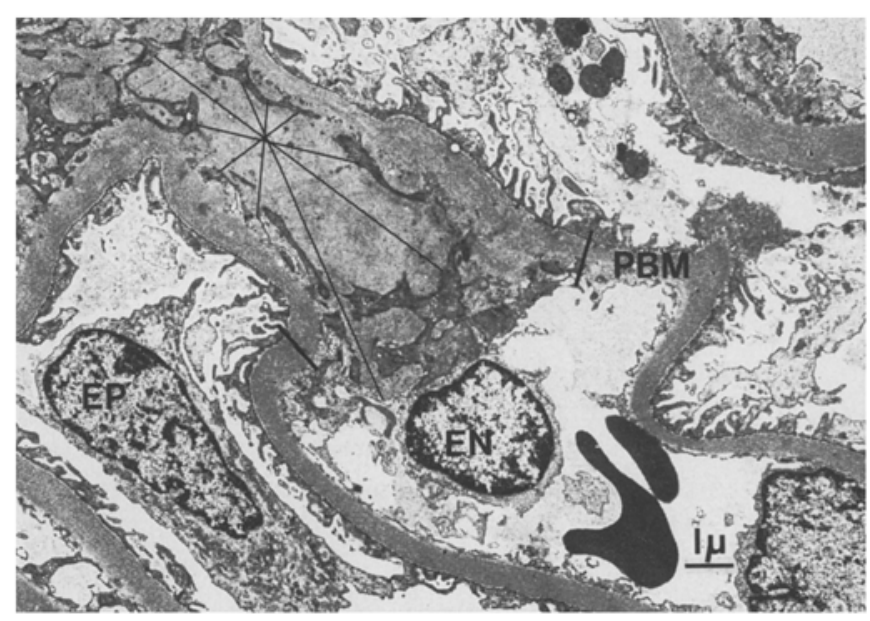

Fig. 1. The micrograph shows a section of a glomerular tuft. The lines separating the peripheral basement membrane (PBM) from the mesangial regions are drawn perpendicular to the epithelial aspect, where the simple three-layered structure (endothelium, basement membrane, epithelium) is separated from the more complex structure of mesangial cells and matrix. The intercepts drawn within one segment of matrix illustrates the matrix star volume, which is the average volume that can be "seen" from random points within the matrix. In the actual measurements intercepts are only classified in one, predetermined direction in each micrograph. EN: endothelial cell, EP: epithelial cell 


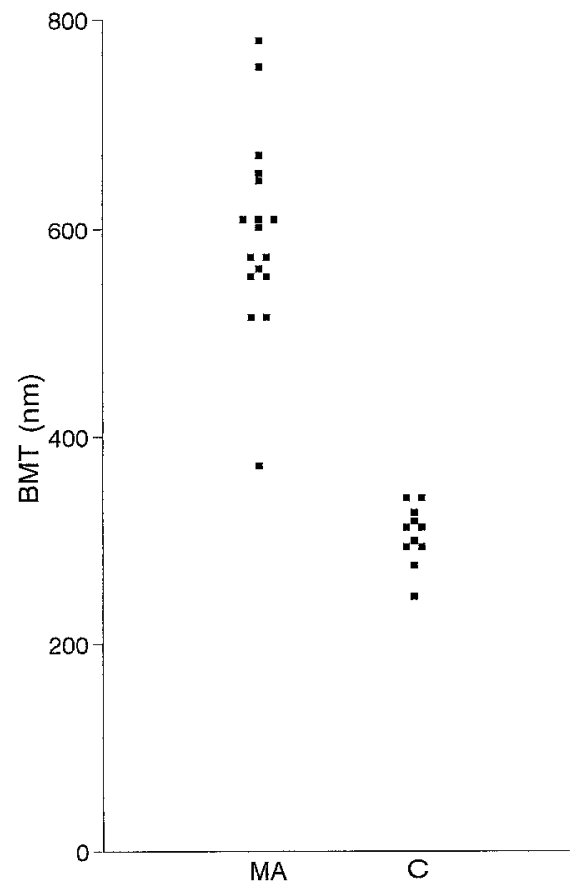

Fig. 2. Basement membrane thickness (BMT) in 17 Type 1 diabetic patients with microalbuminuria (MA) and 11 non-diabetic control subjects (C)

several cases to supplement with extra profiles from other glomeruli that were available on the sections.

The mesangial volume fraction: was estimated by point counting, using an 8: 1 grid, counting hits on mesangium with the fine points, and hits on the reference space, defined as the circumscribed, minimal convex polygon [13] with the coarse points. The polygon, in the following termed 'glomerulus', is the reference space common to light and electron microscopy. Further, coarse points hitting the glomerular tuft, defined as all structures circumscribed by the base of the epithelial foot processes, were counted. The distance between coarse points in the grid corresponded to $22 \mu \mathrm{m}$. The mesangial volume fraction estimate, $V_{v}$ (mes/glom), in each biopsy was obtained as the ratio of sum of points from all profiles hitting mesangium and glomerulus, respectively.

Estimation of surfaces: was done by counting intersections between test lines and the boundaries of the three interfaces: peripheral $\mathrm{BM} /$ urinary space, mesangium/urinary space, and mesangium/capillary space. The surface density of the boundary equals: $2 \cdot$ intersections per test line length within the reference space. The surface density estimate was used for the calculation of the derived structural quantity, matrix-"thickness" (matrix-T), and for the subsets of mesangial surfaces (see below).

From the largest of the three profiles of the level-sectioning a set of micrographs was obtained at a higher magnification $(9990 \times)$, covering about $35 \%$ of the total area. This set of micrographs was placed independently of the structural appearances, the micrographs taken at predetermined settings of the specimen stage movement controls. The set was used for the following estimates:

Thickness of the peripheral basement membrane (BMT): the delineation between peripheral BM and the mesangial matrix was defined as illustrated in Figure 1. Measuring points were sampled independently of the BM appearance with a line grid, measurements taken at intersections between grid lines and the endothelial-BM interface. The 'true BM thickness' was estimated [14], classifying only at the sampling places, where the distinctness of the epithelial cell membrane showed that the section was perpendicular to the BM surface.

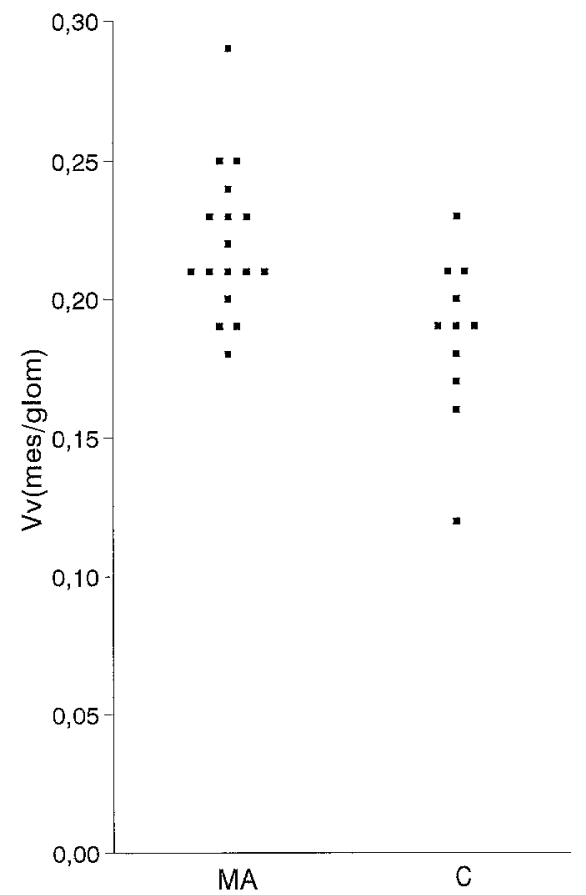

Fig.3. Mesangial volume faction, $\mathrm{V}_{\mathrm{V}}$ (mes/glom), in 17 Type 1 diabetic patients with microalbuminuria (MA) and 11 non-diabetic control subjects (C)

Matrix volume fraction: mesangial matrix was defined as all extracellular material in the mesangial regions. An integral test system was used to count points hitting matrix, mesangial regions, and the glomerular tuft (definition as at low magnification). The grid used for this counting had a $4: 2: 1$ set of points. The $V_{V}$ (matrix/mes) multiplied by $\mathrm{V}_{\mathrm{V}}$ (mes/glom), provided the estimate of the matrix volume fraction, $V_{\mathrm{V}}($ matrix/glom $)$.

Matrix star volume: this estimate was obtained by measuring linear intercepts in the matrix, from random points within matrix, in a direction with a 3-D uniform orientation distribution [15]. This 3-D distribution was obtained by the sampling of sections and micrographs. The intercepts were classified on the micrographs, sampling the measuring points with the point-grid, and measuring in a predetermined direction on the micrograph, i.e. in any direction in 3-D space. The point-sampled intercepts $\left(l_{0}\right)$ were classified with a ruler. All calculations were carricd out on a cubed-scale $\left(1^{3}\right)$. The matrix star-volume, $\mathrm{V}^{*}=\frac{\pi}{3} \cdot\left(\mathrm{l}_{\mathrm{o}}^{3}\right)$.

\section{Derived structural quantities:}

Matrix-T ("thickness"): an estimate of the (imaginary) matrix thickness was obtained from $V_{v}$ (matrix/tuft)/S $($ (matrix/urinary space)/tuft). The dimension of the estimate is $1 / \mu \mathrm{m}^{-1}=\mu \mathrm{m}$, and it corresponds to the thickness of the matrix, if it were spread out in an even layer on the mesangial/urinary space surface.

Mesangial surfaces: the ratio of intersections between grid lines and the mesangial surfaces, the mesangial capillary space and mesangial urinary space interfaces, provided an estimate of the ratio of these two subsets of mesangial surface.

Capillary circumference: was estimated as the ratio of capillary surface density and length density $[16,17]$. This led to an estimate of mean capillary diameter, cap $\mathrm{d}=\mathrm{S}_{\mathrm{V}} / \mathrm{L}_{\mathrm{V}} \cdot \pi$, assuming the capillary walls unfolded. The $S_{\mathrm{V}}$ is the sum of surface density of peripheral BM and the mesangial-capillary interface. The estimate of capillary 


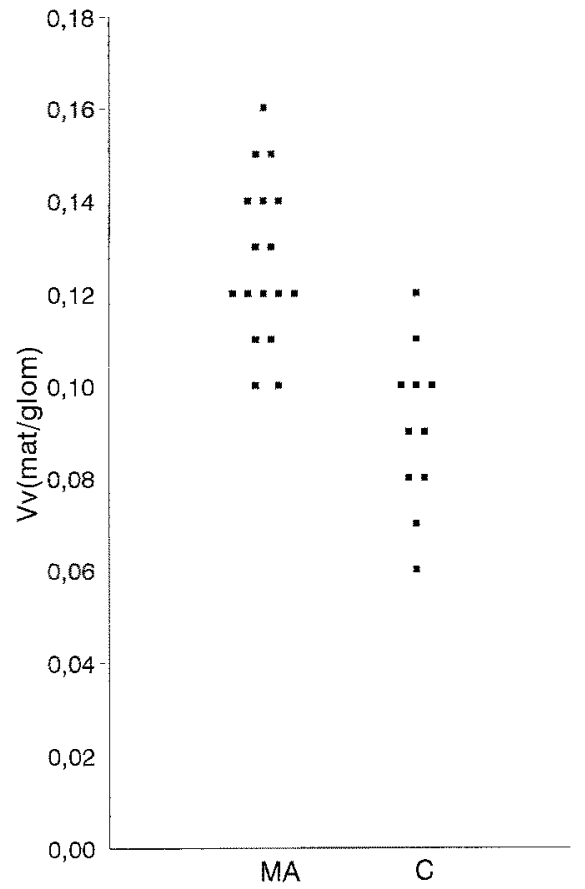

Fig. 4. Matrix volume fraction, $V_{V}$ (mat/glom), in 17 Type 1 patients with microalbuminuria (MA) and 11 non-diabetic control subjects (C)

length density is $L_{V}=2 Q_{A}$, where $Q_{A}$ is the number of capillary profiles per reference area identical to reference area for the $S_{V}$ estimate.

Estimation of interstitial volume fraction by light microscopy: From each biopsy semi-thin plastic sections from three different blocks of tissue were used. The sections at the level $10 \mu \mathrm{m}$ from baseline sections were projected to a television screen at $400 \times$ magnification. The screen was overlaid with a double grid, with coarse and fine points (ratio $4: 30$ ) with distances of $190 \mu \mathrm{m}$ and $70 \mu \mathrm{m}$, respectively. The entire sections were measured, the area studied per biopsy was on average $1.33 \mathrm{~mm}^{2}$ (true area in the kidney). Counting was done of coarse points hitting cortex, and fine points hitting interstitium, glomeruli including Bowman's capsule, and large vessels. The definition of interstitium was the entire space in between tubular epithelium, glomeruli and large vessels, i. e. including interstitial cells and capillaries. The interstitial space was expressed as fraction of cortex minus glomeruli and large vessels, i.e. the "tubular cortex" which is the space where interstitium occurs.

\section{Statistical analysis}

Comparisons between the two groups were done by a one-tailed Student's $t$-test (two-tailed for capillary diameter) and correlations within the diabetic group by least square regression to the mean. Multiple linear regression was used to study the simultaneous effect on structural parameters of various variables. The level of statistical significance was set to $5 \%$.

\section{Results}

The microalbuminuric patients showed a clear increment in BM thickness. BM thickness was significantly increased in the group of microalbuminuric diabetic patients (mean with $95 \%$ confidence intervals): $595 \mathrm{~nm}(549-641)$ compared to the controls $305 \mathrm{~nm}$ (287-325), Figure 2,

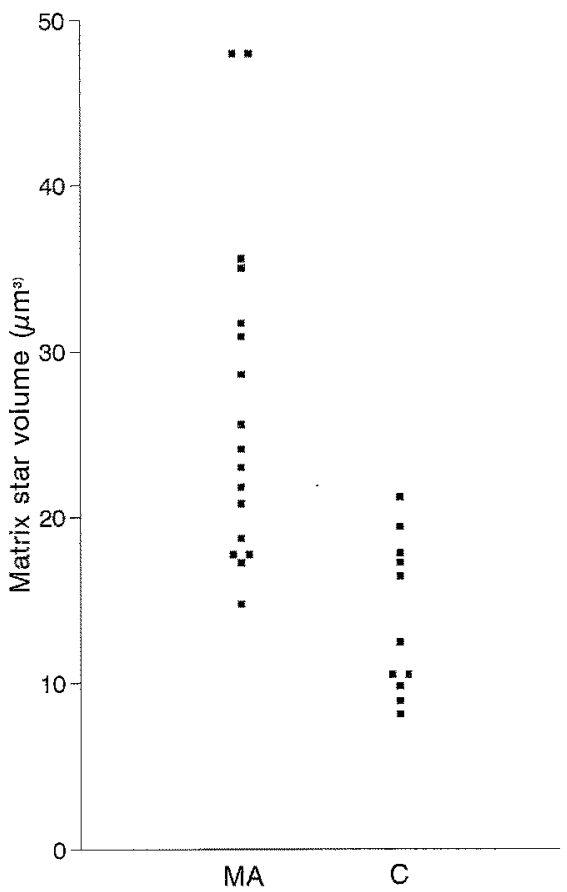

Fig.5. Matrix star volume in 17 Type 1 diabetic patients with microalbuminuria (MA) and 11 non-diabetic control subjects (C)

$(p=0.001)$. All of the diabetic patients had a BM thickness above the normal range.

A significant difference was also found concerning mesangial regions and the mesangial matrix. The difference was most pronounced for the matrix; $\mathrm{V}_{\mathrm{V}}$ (mes/glom) $[0.22$ $(0.21-0.23)$ vs $0.19(0.18-0.21), p=0.04]$, and $V_{\mathrm{V}}(\mathrm{ma}-$ trix/glom) [0.13 (0.12-0.13) vs $0.09(0.08-0.10), p=0.001]$, as seen in Figures 3 and 4 . This indication of changes of mesangial matrix was supported by the two other matrix parameters, matrix star-volume $\left[27.0 \mu \mathrm{m}^{3}(21.8-32.2)\right.$ vs $\left.13.9 \mu^{3}(10.7-17.1), p=0.001\right]$ and matrix-"thickness" $[3.4 \mu \mathrm{m}(2.9-3.8)$ vs $1.5 \mu \mathrm{m}(1.2-1.8), p=0.0001]$, which both differed significantly between the diabetic and normal groups respectively (Figs.5 and 6).

Glomerular capillary diameter was increased in the diabetic group [7.3 $\mu \mathrm{m}(6.9-7.7)$ vs $6.6 \mu \mathrm{m}(6.1-7.1)$, $p=0.01]$. The ratio of the two subsets of mesangial surface, urinary to capillary surface, was reduced in the diabetic group as compared to the control group $[0.70$ $(0.65-0.75)$ vs $1.08(0.91-1.24), p=0.001]$.

Variation between glomeruli was estimated by the intra-biopsy $\mathrm{CV}, \mathrm{CV}=\mathrm{SD} /$ mean, in cases where estimates were obtained in at least three glomeruli. In the diabetic group the $\mathrm{V}_{\mathrm{V}}$ (mes/glom)-variation was estimated only when at least two levels per glomerulus were measured. The mean $\mathrm{CV}$ of the BM thickness and the $\mathrm{V}_{\mathrm{V}}(\mathrm{mes} / \mathrm{glom})$ in the diabetic group were $7.4 \%$ and $11.7 \%$, respectively, In the control group the comparable $\mathrm{CV}$ for BM thickness was $9 \%$. The CV for $\mathrm{V}_{\mathrm{V}}$ (mes/glom) among glomeruli, measured only at one level, was $25 \%$.

The one non-glomerular parameter included, interstitial volume fraction, was increased in the diabetic group as compared to the controls $[0.31(0.27-0.34)$ vs $0.23(0.18$ $0.28), p=0.006]$. 


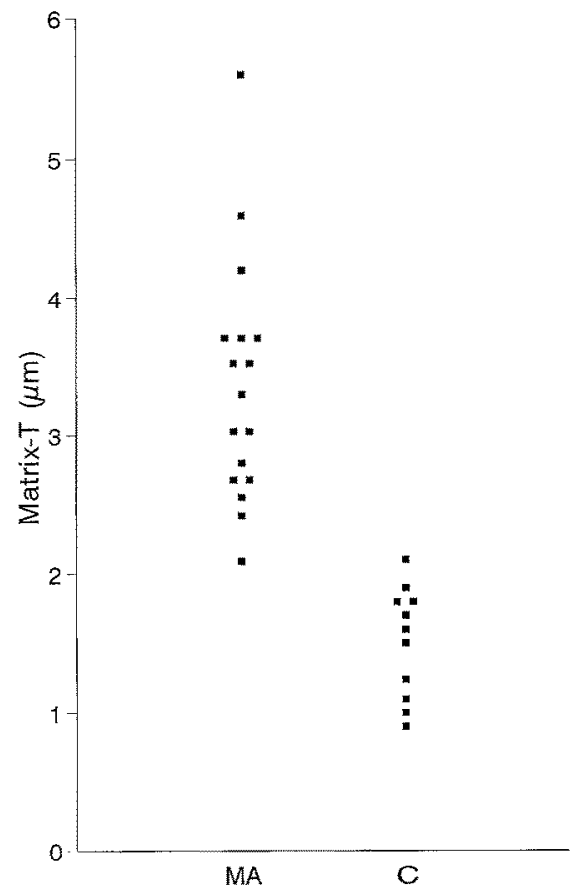

Fig.6. Matrix "thickness" (matrix-T) in 17 Type 1 diabetic patients with microalbuminuria (MA) and 11 non-diabetic control subjects (C)

Within the diabetic group a significant positive correlation $(r=0.67, p=0.02)$ was observed between the overall severity of glomerulopathy, expressed as an index $\left(\mathrm{BMT} / 10+\mathrm{V}_{\mathrm{V}}(\mathrm{mat} / \mathrm{glom}) \cdot 100\right)$ and the interstitial volume fraction. The level of AER did not show a positive correlation with either the severity of glomerulopathy or the interstitial volume fraction. Furthermore, the estimate of mean capillary diameter did not correlate with the current level of GFR $(r=-0.28)$

The relationship between structural changes and putative risk factors, $\mathrm{HbA}_{1 \mathrm{c}}$, systolic and diastolic blood pressure, and the level of GFR, were studied. In these correlations we used an estimate of structural change per year of diabetes, taking the mean value for the control group as the best bid on baseline value, i. e. $305 \mathrm{~nm}$ for BM thickness and 0.09 for $\mathrm{V}_{\mathrm{v}}$ (mat/glom). The results of these calculations (Table 2) showed a positive correlation between mean-one year $\mathrm{HbA}_{1 \mathrm{c}}$ and both $\mathrm{BM}$ thickening (Fig. 7) and increment in $\mathrm{V}_{\mathrm{v}}$ (matrix/glom), $r=0.55(p=0.02)$ and $r=0.51 \quad(p=0.02)$, respectively. When we applied multiple linear regression analysis with these two structural parameters separately as dependent variables and

Table 2. Correlation coefficient $(r)$ between clinical variables and estimated rate of increment in structural parameters

\begin{tabular}{|c|c|c|}
\hline & $\begin{array}{l}\text { Basement membrane } \\
\text { thickening }{ }^{2}\end{array}$ & $\begin{array}{l}V_{v}(\text { mat/glom }) \\
\text {-increment }\end{array}$ \\
\hline $\mathrm{Hb}_{1 \mathrm{c}}$ (mean one-year) & 0.55 & 0.51 \\
\hline Systolic blood pressure & 0.15 & 0.01 \\
\hline Diastolic blood pressure & 0.24 & 0.08 \\
\hline Glomerular filtration rate & 0.12 & $\div 0.18$ \\
\hline
\end{tabular}

a (Basement membrane thickness $-305 \mathrm{~nm}$ )/duration of diabetes;

${ }^{\mathrm{b}}\left(\mathrm{V}_{\mathrm{V}}\right.$ mat/glom -0.09$)$ /duration of diabetes

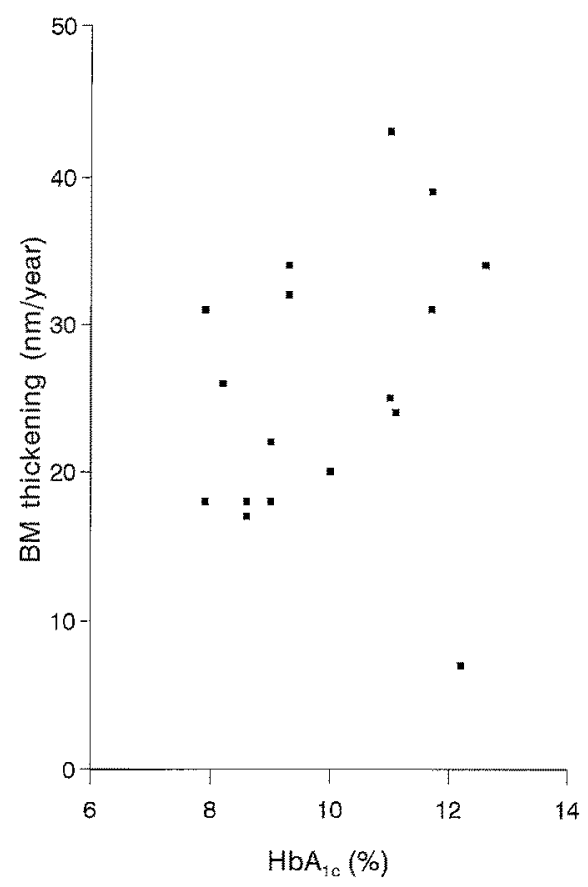

Fig. 7. Basement membrane (BM) thickening per year [(basement membrane thickness $-305 \mathrm{~nm} /$ duration of diabetes in years] measured in 17 Type 1 diabetic patients vs mean 1-year $\mathrm{HbA}_{1 \mathrm{c}} .305$ $\mathrm{nm}=$ mean value of the control group

$\mathrm{HbA}_{1 \mathrm{c}}$, GFR, duration of diabetes, systolic and diastolic blood pressure as independent variables, we confirmed a significant correlation with $\mathrm{HbA}_{1 \mathrm{c}}(p<0.05)$, but with none of the other variables. One outlier with BM thickness of $372 \mathrm{~nm}$ was excluded from the calculation of the correlations. The BM in this biopsy was atypical with varying thickness and fluffy areas.

\section{Discussion}

We have demonstrated distinct morphological changes in the $B M$ and the mesangium, corresponding to an early stage of diabetic glomerulopathy in a group consisting of 17 young, Type 1 diabetic patients characterized by normal blood pressure (except for one patient), slightly elevated albumin excretion and glomerular hyperfiltration. For BM thickness it is noteworthy that there was no overlap between the findings in the diabetic and the nondiabetic control group.

The increment of the matrix parameters was clearly present and the study showed that structural changes do exist, not only in the BM thickness, but also in parameters reflecting mesangial matrix expansion in patients with microalbuminuria. The elaborate protocol using more than the one level per glomerulus led to low intra-biopsy variation, which was essential for demonstration of the modest mesangial changes.

The reference group differed from the diabetic group with respect to age, sex and GFR. However, we saw no relationship between BM thickness and $V_{v}$ (mes/glom) vs age in the donor kidneys, observations similar to those reported in another series of non-diabetic patients [18]. Fur- 
thermore, due to older age in the control group any bias would be towards more pathology.

The protocol used for the reference group was less extensive than that used for the diabetic patients. This concerned the mesangial estimates (with the exception of the matrix star volume) and those of the surfaces. The higher $\mathrm{CV}$ in the control group of mesangial and matrix parameters is likely to reflect this difference in the protocol. With the sampling of three levels per glomerulus for the mesangial estimates, intra- as well as inter-biopsy variation in the diabetic group was very low. Also the BM thickness estimates showed a low intra-biopsy variation. These findings therefore indicate that in early diabetic glomerulopathy the lesions are very uniformly distributed within the kidney, or at least within the space of a kidney biopsy.

It has been shown that newly-diagnosed diabetic patients $[14,19]$ and identical non-diabetic twins of diabetic patients [20] reveal normal kidney morphology. Structural studies in the clinically silent phase, $i$. e. before the onset of microalbuminuria, are scarce. It has been demonstrated that BM thickening may be present after a few years of diabetes, both in native kidneys [14] or in the transplanted kidney $[21,22]$. This might be an indication that the findings in the present series are unrelated to the presence of microalbuminuria. Our study design did not include diabetic patients with normoalbuminuria. Biopsies from patients of this category, comparable with our group of microalbuminuric patients with respect to important factors such as diabetes duration and GFR, have recently been investigated in the same laboratory as our biopsies [8]. Comparing the data from our group of microalbuminuric patients with those in the published series of patients with normoalbuminuria, shows significant differences (mean) in BM thickness ( $595 \mathrm{~nm}$ vs $442 \mathrm{~nm}$ ), as well as the matrix-parameters [matrix star volume $27 \mu \mathrm{m}^{3}$ vs $22 \mu^{3}$; matrix-T $3.4 \mu \mathrm{m}$ vs $2.2 \mu \mathrm{m}$; $V_{\mathrm{v}}$ (matrix/glom) 0.13 vs 0.11 ], whereas the mesangial volume fraction is at a similar level in the two groups $(0.22$ vs 0.22$)$. The mesangial volume fraction in the present series showed only borderline increase as compared to the control groups. Thus, the expansion of the mesangial regions is not marked at this stage although pooled data from different series, all of them studied at the same laboratory [9], reveals significant differences between patients with normo- and microalbuminuria also concerning the mesangial volume fraction (0.22 vs 0.26$)$.

The matrix parameters, star volume and thickness, confirmed that matrix changes are present at this early stage. The star volume expresses the degree of matrix confluence, or, in other words, the separation of individual segments of mesangial cytoplasm. The concurrent changes in the ratio of the two subsets of mesangial surfaces, the entry-side being larger than the exit-side, might favour accumulation of blood-borne substances within the mesangial regions. Thus the structural modifications which we have demonstrated may tend to interfere with the mesangial cell function, e.g. the turn-over of extracellular material [23], regulation of intraglomerular haemodynamics [24], and interference with the peripheral filter [25]. The microalbuminuria is the clinical sign that the filter does not function normally.
In the study reported by Chavers et al. [5] no significant difference was found between microalbuminuric patients with normal blood pressure and preserved GFR vs normoalbuminuric diabetic patients, whereas our microalbuminuric group showed both BM thickness- and mesangial matrix-differences when compared to the previously published normoalbuminuric group [8]. This may reflect the large inter-individual CV $(0.33)$ in mesangial volume fraction in the study [5]. Furthermore, the definition of microalbuminuria was based on only one urine sample in some of the patients. Therefore, the criteria for persistent microalbuminuria were not fulfilled.

The link between progressing glomerulopathy and increased AER has not been identified. We found no significant correlation between the glomerular parameters and AER. However, the AER in our series was in most cases in the low microalbuminuric range.

It seems natural to associate the finding of increased capillary diameter with the hyperfiltration state in the diabetic group. However, no correlation between current level of GFR and the mean diameter was observed. Whether the increase in diameter reflects an increase in glomerular volume at this stage of disease has not yet been determined. The variation among patients was very low $(\mathrm{CV}=0.10)$, most likely depending on the use of multiple profiles for the estimate.

The estimate of interstitial volume fraction was obtained from a limited area. Thus, the finding of increment compared to the control group, must be interpreted with caution. In renal disorders showing interstitial fibrosis the lesions usually are patchy. Furthermore, the presence or absence of large blood vessels in the tissue used for measurements could affect the estimate obtained from areas of limited size. We chose to express the interstitial tissue as a fraction of the "tubular cortex" in order to minimize this influence. Accumulation of interstitial tissue is always present around the larger blood vessels and immediately surrounding the glomeruli. It is therefore important that in the present series no correlation was found between the interstitial volume fraction and the area of large vessels or glomeruli in the sections under study (data not shown). The finding of increased interstitial volume fraction in the present kidney biopsies seems to indicate that the processes occurring in the glomeruli and in the interstitium takes place in parallel.

An association between the severity of long-term hyperglycaemia and renal morphological changes has not previously been demonstrated. We found that mean oneyear $\mathrm{HbA}_{1 \mathrm{c}}$ was positively correlated to an estimated rate of BM-thickening and matrix volume-fraction increment. Ideally the mean $\mathrm{HbA}_{1 \mathrm{c}}$ from the time of diagnosis of diabetes should have been studied, but these data are not available for all patients. Our clinical experience is that glycated haemoglobin measured during 1 year in most patients is representative of their long-term glycaemic level. The lack of correlation between other putative risk factors and the pathological changes may of course simply express a statistical type 2-error. The absent correlations do not exclude that either of these clinical variables may influence the development of renal lesions in diabetes. On the other hand, the observations do not give any support 
to the presumed influence of hyperfiltration or elevated systemic blood pressure in initiating the lesions of diabetic glomerulopathy.

Even though microalbuminuria predicted clinical diabetic nephropathy [1-3], it was found in a recent 4-year follow-up study [26] that $50 \%$ of the insulin-dependent diabetic patients with microalbuminuria did not show an increase of urinary AER over that period. The individual variation in the pattern of development of the structural changes over the duration of diabetes is unknown. Whether some patients may develop only a degree of glomerulopathy and then show no further progression due to intervention treatment or spontaneously - can only be elucidated in a follow-up series. The present series represents baseline values for such a follow-up study.

Our studies emphasize the importance of detecting microalbuminuria and following the course of development of nephropathy in young diabetic patients.

Acknowledgements. The skillful technical assistance of Ms K. Gerlach, Ms B. Saugbjerg, Ms B. Iversen and Ms J. Arve is gratefully acknowledged. The study was supported by grants from the following: Norwegian Diabetes Association, Jahre's Foundation, Norwegian Medical Research Council (NAVF), Odd Fellows Medical Founda tion, Novo-Nordisk Pharma, Norwegian Hoechst A/S, Juvenile Diabetes Foundation Int., grant \# 190592, The Danish Diabetes Association, The Danish Medical Research Council, Lagevidenskabens Fremme, Bernhard and Marie Klein's Legat, Ruth IE Konig-Petersens's Fond, NOVO Foundation, Århus University Research Foundation.

\section{References}

1. Viberti GC, Hill RD, Jarrett RJ, Argyropoulos A, Mahmud U, Keen H (1982) Microalbuminuria is a predictor of clinical nephropathy in insulin-dependent diabetes mellitus. Lancet I: 1430-1432

2. Parving HH, Oxenbøll B, Svendsen PAa, Sandahl Christiansen J, Andersen AR (1982) Early detection of patients at risk of developing diabetic nephropathy. A longitudinal study of urinary albumin excretion. Acta Endocrinol 100: 500-505

3. Mogensen CE (1987) Microalbuminuria is a predictor of clinical diabetic nephropathy. Kidney Int 31: 673-689

4. Deckert T, Feldt-Rasmussen B, Borch-Johnsen K, Kofoed-Enevoldsen A (1989) Albuminuria reflects widespread vascular damage. The Steno hypothesis. Diabetologia 32: 219-226

5. Chavers MB, Bilous RW, Ellis EN, Steffes MW, Mauer SM (1989) Glomerular lesions and urinary albumin excretion in type I diabetes without overt proteinuria. $N$ Engl J Med 320: 966-970

6. Østerby R, Andersen AR, Gundersen HJG, Jørgensen HE, Mogensen CE, Parving H-H (1984) Quantitative studies of glomerular ultra structure in type 1 diabetics with incipient nephropathy. Diabetic Nephropathy 3:95-100

7. Inomata S, Oosawa Y, Itoh M, Masamune O (1987) Analysis of urinary proteins in diabetes mellitus - with reference to the relationship between microalbuminuria and diabetic lesions. J Jpn Diabetes Soc 30: 429-435

8. Walker JD, Close CF, Jones SL et al. (1992) Glomerular structure in type-1 (insulin-dependent) diabetic patients with normo and microalbuminuria. Kidney Int 41: 741-748
9. Østerby R (1992) Glomerular structural changes in type 1 (insulin-dependent) diabetes. Consequences, causes, and prevention. Diabetologia 35: 803-812

10. Nyberg G, Hedman L, Blohme I, Svalander C (1992) Morphological findings in baseline kidney biopsies from living related donors. Transpl Proc 24: 355-356

11. Brøchner-Mortensen J (1972) A simple method for the determination of glomerular filtration rate. Scand J Clin Invest 30:271274

12. Maunsbach $\mathrm{AB}$ (1966) The influence of different fixatives and fixation methods on the ultrastructure of rat kidney proximal tubule cells. II. Effects of varying osmolality, ionic strength, buffer system and fixative concentration of glutaraldehyde solutions. J Ultrastruc Res 15: 283-309

13. Østerby R, Parving H-H, Nyberg G et al.(1988) A strong correlation between glomerular filtration rate and filtration surface in diabetic nephropathy. Diabetologia 31: 265-270

14. Østerby R (1973) Morphometric studies of the peripheral glomerular basement membrane in early juvenile diabetes II. Topography of the initial lesions. Diabetologia 9: 108-114

15. Gundersen HJG, Bendtsen TF, Korbo L et al. (1989) Some new simple and efficient stereological methods and their use in pathological research and diagnosis. APMIS 96: 379-394

16. Ellis EN, Mauer SM, Sutherland DER, Steffes MW (1989) Glomerular capillary morphology in normal humans. Lab Invest 60: $231-236$

17. Ellis EN, Steffes MW, Goetz FC, Sutherland DE, Mauer SM (1986) Glomerular filtration surface in type 1 diabetes mellitus. Kidney Int 29: 889-894

18. Steffes MW, Barbosa J, Basgen JM, Sutherland DER, Najarian JS, Mauer SM (1983) Quantitative glomerular morphology of the normal human kidney. Lab Invest 49: 82-85

19. Østerby R (1973) A quantitative electron microscopic study of mesangial regions in glomeruli from patients with short term juvenile diabetes mellitus. Lab Invest 29: 99-110

20. Steffes MW, Sutherland DER, Goetz FC, Rich SS, Mauer SM (1985) Studies of kidney and muscle biopsies in identical twins discordant for type I diabetes mellitus. N Engl J Med 312: 12811287

21. Mauer SM, Goetz FC, McHugh LE et al. (1989) Long-term study of normal kidneys transplanted into patients with type 1 diabetes. Diabetes 38:516-523

22. Østerby R, Nyberg G, Hedman L, Karlberg I, Persson H, Svalander C (1991) Kidney transplantation in type 1 (insulin-dependent) diabetic patients. Early glomerulopathy. Diabetologia 34: 668-674

23. Kanwar YS (1984) Biology of disease. Biophysiology of glomerular filtration and proteinuria. Lab Invest 5: 7-21

24. Sakai T, Kriz W (1987) The structural relationship between mesangial cells and basement membrane of the renal glomerulus. Anat Embryol 176: 373-385

25. Michael AF, Keane WF, Raij L, Vernier RL, Mauer SM (1980) The glomerular mesangium. Kidney Int 17:141-154

26. Mathiesen ER, Hommel E, Giese J, Parving HH (1991) Efficacy of captopril in postponing nephropathy in normotensive insulin dependent diabetic patients with microalbuminuria. Br Med J 303: $81-87$

Received: 3 December 1992

and in revised form: 29 January 1993

Dr. H.m. Bangstad

Paediatric Department

Aker University Hospital

N-0514 Oslo

Norway 